\title{
Spin-Polarization Measurements in the Autoionization Region of Mercury*
}

\author{
M. Müller, F. Schäfers ${ }^{\dagger}$, N. Böwering, Ch. Heckenkamp ${ }^{\ddagger}$ and U. Heinzmann \\ Fakultät für Physik der Universität Bielefeld, D-4800 Bielefeld 1, Fritz-Haber-Institut der MPG, Faradayweg 4-6, D-1000 Berlin 33
}

Received August 8, 1986; accepted September 30, 1986

\begin{abstract}
The autoionization region of mercury was investigated with spin- and angleresolved photoelectron spectroscopy between the $\mathrm{Hg}^{+}\left(5 d^{9} 6 s^{2}\right)^{2} D_{5 / 2}$ and $\mathrm{Hg}^{+}\left(5 d^{9} 6 s^{2}\right)^{2} D_{3 / 2}$ thresholds. Spin-polarization parameters $A$ and $\xi$ and intensity were measured separately for the photoelectrons corresponding to the two final ionic states accessible $\left({ }^{2} S_{1 / 2},{ }^{2} D_{5 / 2}\right)$ as function of the radiation wavelength. The data are dominated by structure due to autoionization resonances and differ quantitatively for the two peaks in the photoelectron spectra. The results are compared with earlier measurements below the ${ }^{2} D_{s / 2}$-threshold.
\end{abstract}

\section{Introduction}

Starting from the first ionization threshold $\left({ }^{2} S_{1 / 2}\right)$ at $10.4 \mathrm{eV}$ photon energy, the photoionization of mercury $\left(5 d^{10} 6 s^{2}\right)^{1} S_{0}$ is strongly influenced by autoionizing levels of several Rydberg series. These autoionizing levels are characterized by an excitation of a $5 d$-electron and converge to the $D$-threshold which is split by fine structure to a ${ }^{2} D_{5 / 2}$ and a ${ }^{2} D_{3 / 2}$-final ionic state (at $14.8 \mathrm{eV}$ and $16.7 \mathrm{eV}$, respectively).

Following the early work of Beutler [1], the autoionization region of mercury has been investigated by several groups in the last two decades: The photoabsorption experiments of Garton and Connerade [2] and Mansfield [3] established the classification of the resonances. The relative photoionization cross section was measured by Brehm [4], Cairns et al. [5] and scaled absolutely by Berkowitz [6].

Experiments using photoelectron spectroscopy were performed by Blake [7] to separate the two final ionic states and Brehm and Höfler [8] who measured the angular asymmetry parameter $\beta$. Later, Schäfers et al. [9] and Schönhense et al. [10] determined the spin-polarization parameters in the resonance region below the ${ }^{2} D_{5 / 2}$-threshold. In combination with recent high resolution photoabsorption measurements a connection between the Rydberg series below and above the first threshold could be established and a full characterization of the photoionization process with respect to transition-matrix elements and phase-shift differences was possible $[9,10]$. A recent calculation of Bartschat and Scott [11,12] in R-Matrix theory yielded good agreement with the experimental data. It is the purpose of this paper to extend the measurements to the region between the ${ }^{2} D$-thresholds. There not only higher members of the same autoionizing series ( $5 d \rightarrow n p$ excitation) can be investigated, but also another series with a $5 d \rightarrow n f$

\footnotetext{
* This paper was contributed to the 8th Vacuum Ultraviolet Radiation Physics International Conference, held in Lund, Sweden, 4-8 August, 1986, and will be included in part II of the Conference proceedings. (Editors: P. -O. Nilsson and J. Nordgren).

$\dagger$ BESSY, Lentzeallee 100, D-1000 Berlin 33, West Germany

$\$$ Gesellschaft für Automation und Organisation, Euckenstr. 12, D-8000 München 70, W. Germany
}

excitation. However, no theoretical calculation is available for this region.

While the levels below the ${ }^{2} D_{5 ; 2}$ threshold can couple only with the continua corresponding to the ${ }^{2} S_{1 / 2}$ final ionic state, the region between the ${ }^{2} D$-thresholds is characterized by autoionizing levels which interact with the partial continua of two final ionic states, $\mathrm{Hg}^{+2} S_{1 / 2}$ and $\mathrm{Hg}^{+2} D_{5 / 2}$, giving rise to photoelectrons with different kinetic energy $(\Delta E=4.4 \mathrm{eV})$. Therefore, for a detailed analysis in this region, energy-resolved photoelectron-emission studies are required. The results of Blake [7] for the relative cross section demonstrate, that the interaction of the excited states with the continuum states is different for both ionic final states.

In our work of spin-, angle- and energy-resolved photoelectron spectroscopy using circularly polarized light these levels are an interesting subject for further investigation.

\section{Experimental set-up}

The experimental arrangement for the measurements of the spin-polarization parameters at the electron storage ring BESSY (Berlin) was described in detail by Heckenkamp et al. [13-15]. Briefly, monochromatic circularly polarized vuv synchrotron radiation from the $6.5 \mathrm{~m}$-normal-incidencemonochromator [16] is crossed by an atomic beam of mercury which emerges from a resistively heated oven. The photoelectrons emitted in the reaction plane at a definite angle $\theta$ are energy-analyzed by a rotatable hemispherical spectrometer (rotatable around the normal of the reaction plane), and, after two deflections by 90 degrees the electron beam is accelerated to $120 \mathrm{keV}$ and scattered at a thin gold foil of a Mott detector. With the Mott detector two transverse electron spin-polarization components, $A(\theta)$ (component in the direction of the incident light) and $P_{\perp}(\theta)$ (component perpendicular to the reaction plane), are measured simultaneously.

\section{Results and discussion}

The process examined is described by the following scheme (assignments adopted from Garton and Connerade [2]):

$$
\begin{aligned}
& \mathrm{Hg}\left(5 d^{10} 6 s^{2}\right){ }^{1} S_{0} \\
&+h v \underset{\mathrm{Hg}}{\mathrm{Hg}\left[\left(5 d^{9} 6 s^{2}\right)^{2} D_{3 / 2} n p_{1 / 2}, n p_{3 / 2}, n^{\prime} f_{5 / 2}\right]_{J=1}} \\
& \mathrm{Hg}\left[\left(5 d^{10} 6 s\right)^{2} S_{1 / 2} \varepsilon p_{1 / 2}, \varepsilon p_{3 / 2}\right]_{J=1}
\end{aligned}
$$

where $n \geqq 7$ and $n^{\prime} \geqq 5$ (the autoionizing level of $n=6$ lies below the ${ }^{2} D_{5 / 2}$-threshold and was subject of the earlier studies $[9,10])$. 


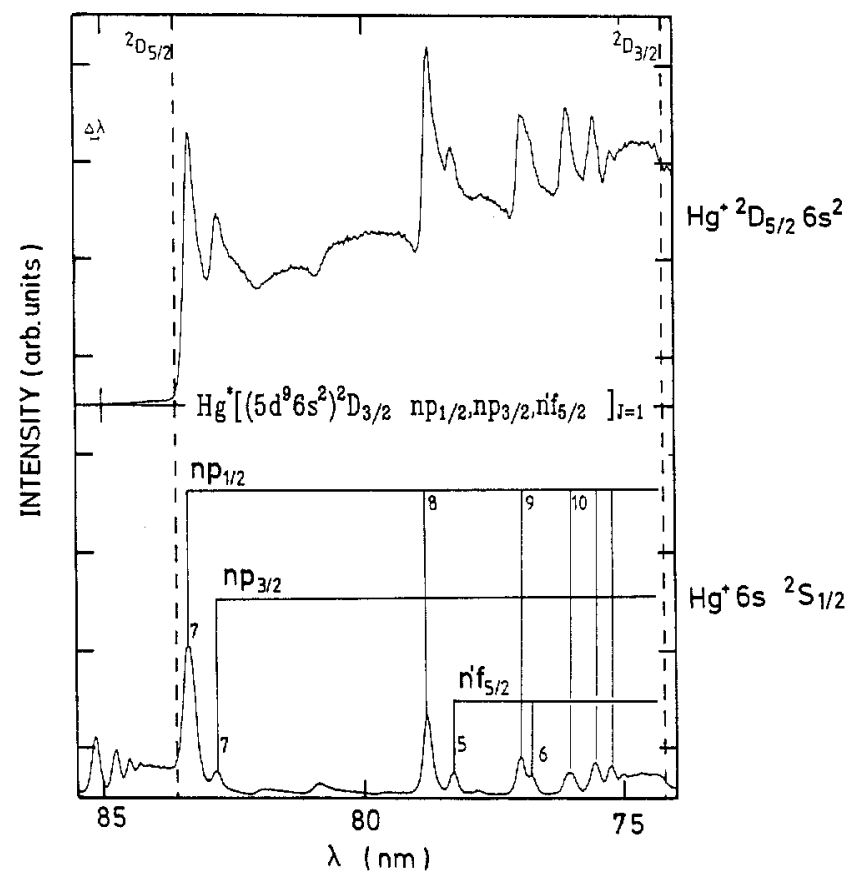

$$
\begin{aligned}
& {\left[\mathrm{Hg}\left(5 \mathrm{~d}^{10} 6 s\right)^{2} S_{1 / 2}+e^{-}\left(\in \mathrm{p}_{1 / 2}, \epsilon \mathrm{p}_{3 / 2}\right)\right]_{\mathrm{J}=1}} \\
& {\left[\mathrm{Hg}\left(5 \mathrm{~d}^{9} 6 \mathrm{~s}^{2}\right)^{2} \mathrm{D}_{5 / 2}+\mathrm{e}^{-}\left(\epsilon \mathrm{p}_{3 / 2}, \epsilon \mathrm{\epsilon}_{5 / 2}, \epsilon \mathrm{f}_{7 / 2}\right)\right]_{\mathrm{J}=1}}
\end{aligned}
$$

Fig. 1. Photoelectron intensity for the two final ionic states: $\mathrm{Hg}^{+} S_{1 / 2}$ (bottom) and $\mathrm{Hg}^{+2} D_{5 / 2}$ (top). The vertical dashed lines indicate the ${ }^{2} D_{5 / 2}{ }^{-}$ and ${ }^{2} D_{3 / 2}$-threshold, respectively.

Fig. 1 shows the measured photoelectron intensities of the two peaks in the spectra $(\Delta E=4.4 \mathrm{eV})$ corresponding to the ${ }^{2} S_{1 / 2}$ (lower half) and the ${ }^{2} D_{5 / 2}$ (upper half) ionic state between the ${ }^{2} D_{5 / 2}$ - and ${ }^{2} D_{3 / 2}$-thresholds. According to photoabsorption measurements [2] there are three Rydberg series converging to the ${ }^{2} D_{3 / 2}$-threshold, namely the ${ }^{2} D_{3 / 2} n p_{1 / 2}, n p_{3 / 2}$ and $n^{\prime} f_{5 / 2}$ series. In the case of the $\mathrm{Hg}^{+2} D_{5 / 2}$ final ionic state the resonances are superimposed on a large non-interacting background continuum, which due to a potential barrier effect is increasing towards higher photon energies. The lower part of Fig. 1 shows the corresponding spectrum for the $\mathrm{Hg}^{+}{ }^{2} S_{1 / 2}$ final ionic state. In this case the direct photoionization process is very weak, and the cross section is governed predominantly by the resonances. From the line profiles of these resonances one can deduce that the shapes differ significantly for both final ionic states. The $\mathrm{Hg}^{+2} D_{5 / 2}$ case exhibits strongly asymmetric Fano-profiles [17], whereas the profiles corresponding to the ${ }^{2} S_{1 / 2}$ ionic state show almost Lorentzian shape with small asymmetry.

An interesting feature is observed between the $n=7$ and $n=8$ resonances at $\lambda \simeq 81 \mathrm{~nm}$ and $\lambda \simeq 82 \mathrm{~nm}$ : weak structures occur which give rise to "window resonances" [17] in the ${ }^{2} D_{5 / 2}$ channel (Fig. 1 upper part), whereas in the ${ }^{2} S_{1 / 2}$ channel (lower part of Fig. 1) broad asymmetric profiles appear. In photoabsorption measurements, which correspond to the sum of our photoelectron spectra, these resonances should be masked because their respective contributions cancel. A similar behavior has been observed recently for an autoionizing level of lead by Krause et al. [18]. Work to resolve the origin of these two new resonances and their classification is still in progress.

Fig. 2 shows the spin polarization parameter $A(=A(\theta)$ at
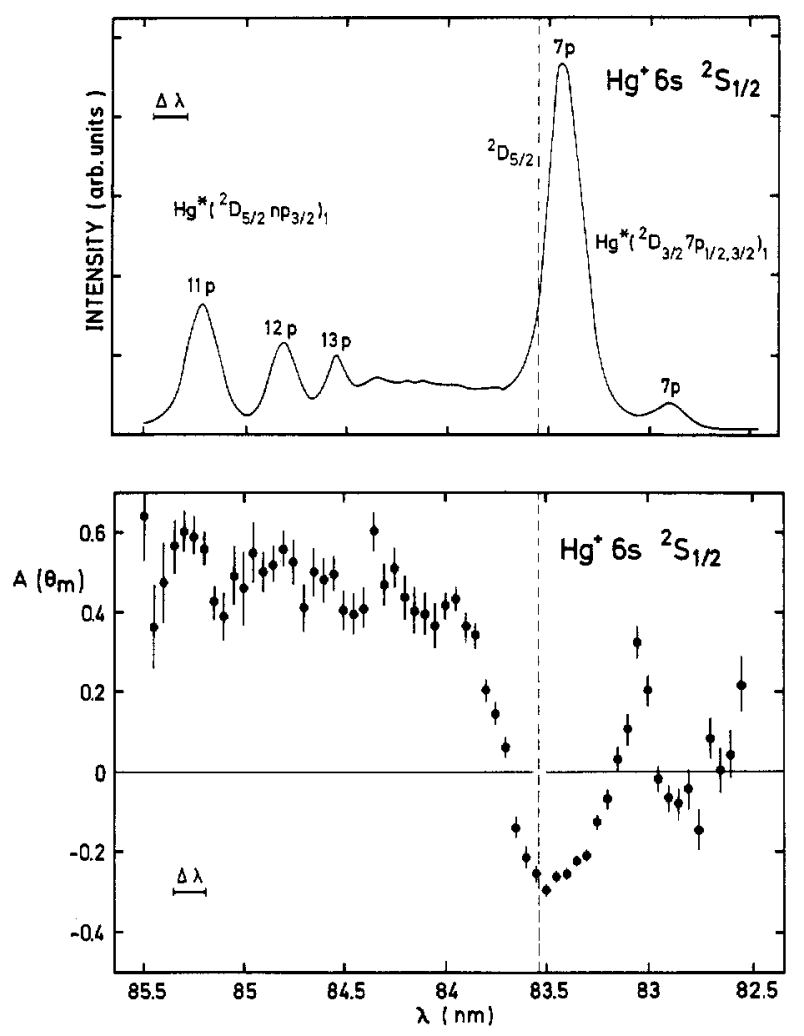

Fig. 2. Spin-polarization parameter $A=A\left(\theta_{\mathrm{m}}\right)$ (bottom) and photoelectron intensity (top) for the ${ }^{2} S_{1 / 2}$ ionic state in the region of the ${ }^{2} D_{5 / 2}$-threshold.

the magic angle $\theta_{\mathrm{m}}=54^{\circ} 44^{\prime}$ ) and the corresponding photoelectron intensity (for the ${ }^{2} S_{1,2}$-ionic state) for the first two resonances above the ${ }^{2} D_{5 / 2}$-threshold $(n=7)$ and for higher members of the Rydberg series (partially resolved) converging to the ${ }^{2} D_{5 / 2}$-threshold. The autoionizing resonances have a dominating influence on the spin-polarization data. The polarization in the first resonance $\mathrm{Hg}^{* 2} D_{3 / 2} 7 p_{1 / 2}$ varies from $+40 \%$ to $-30 \%$, whereas in the second resonance $\mathrm{Hg}^{*}$ ${ }^{2} D_{3 / 2} 7 p_{3 / 2}$ a sharp change of sign occurs. These results are similar to earlier data measured for the $n=6$ member of the same series [9] where no energy analysis of the photoelectrons had been performed. Due to our lower wavelength resolution $(\Delta \lambda=0.2 \mathrm{~nm})$ the maxima of the polarization curve are lower than for the $n=6$ case [9]. Spin-polarization measurements for the other ionic final state could not be performed since the signal is masked by the background in the spectrometer at low kinetic energies ( $\leqq 100 \mathrm{meV})$. Further investigations concentrated on the next numbers of the Rydberg series $8 p_{1 / 2}, p_{3 / 2}$ (not resolved) and on the $5 f_{5 / 2}$ resonance. In Fig. 3 and 4 the spin-polarization parameters $A=A(\theta)_{\mathrm{m}}$ and $\xi$ (describing the component $P_{\perp}(\theta)$ normal to the reaction plane) are shown for both ionic states. In the case of the ${ }^{2} S_{1 / 2}$-ionic channel (Fig. 3) the $p$-resonance appears as a deep minimum in both polarization parameters $A$ and $\xi$, as was the case at the $7 p_{3 / 2}$ and $7 p_{1 / 2}$ (Fig. 2) resonances of the same Rydberg series, indicating a similar coupling to the two possible partial continua $\varepsilon p_{3 / 2}$ and $\varepsilon p_{1 / 2}$. From the behavior of the $\xi$ parameter we deduce a change of sign of the phase-shift difference at the $\xi=0$ positions. The $f$-resonance is characterized by a broad structure with large positive values of the polarization parameter $A$.

As seen in Fig. 1 the autoionization resonances for the ${ }^{2} D_{5 / 2}$ case are superposed onto a large non-interacting con- 


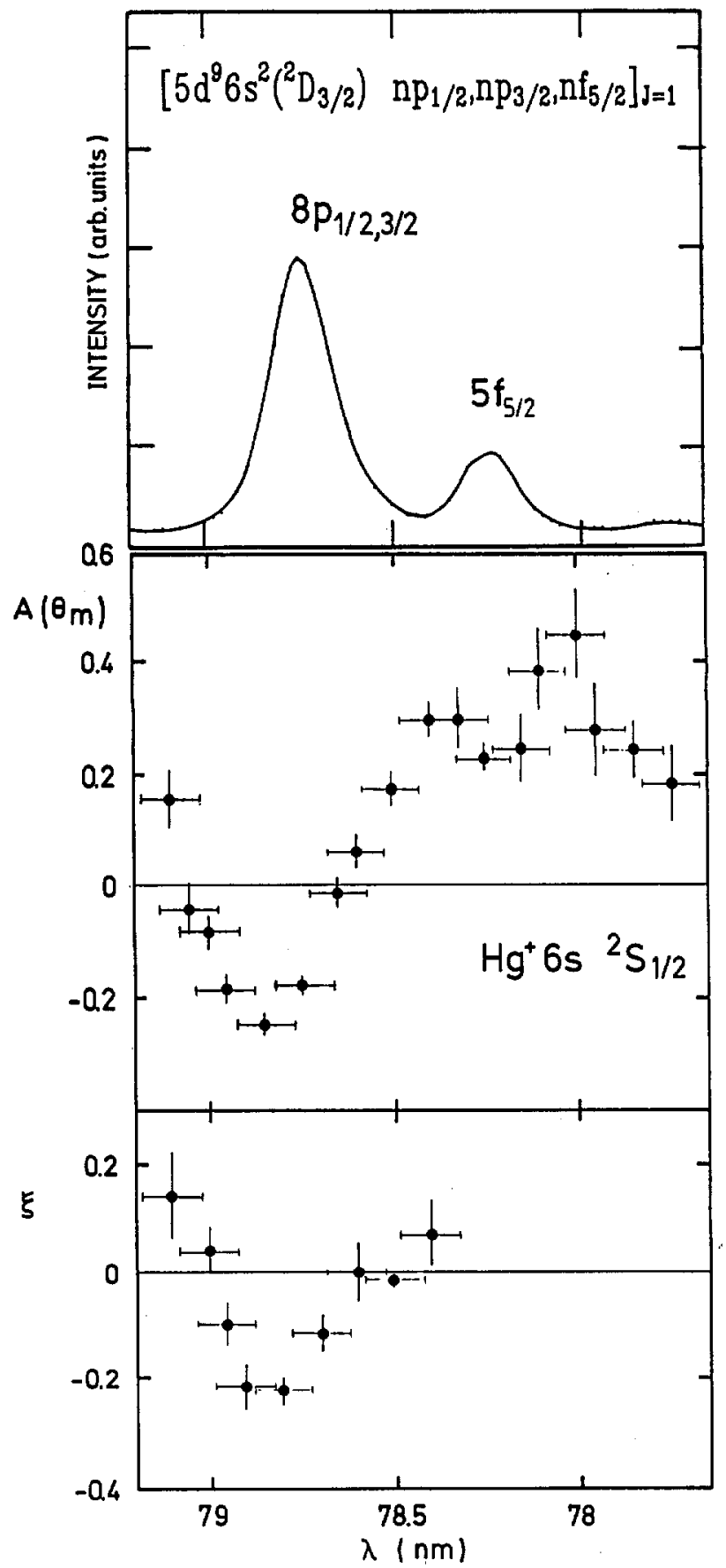

Fig. 3. Photoelectron intensity and spin-polarization parameters $A$ and $\xi$ as a function of the wavelength in the range of the $\mathrm{Hg}^{*}\left(5 d^{9} 6 s^{2}\right)^{2} D_{3 / 2} 8 p_{1 / 2}, p_{3 / 2}$ and $5 f_{5 / 2}$ resonance for $\mathrm{Hg}^{+}{ }^{2} S_{1 / 2}$.

tinuum. The influence on the photoelectron polarization is therefore expected to be less pronounced. This is demonstrated in Fig. 4 in the case of the p-resonance. While the shape of the wavelength dependence of $A\left(\theta_{\mathrm{m}}\right)$ is similar, the absolute values are much smaller than for the corresponding ${ }^{2} S_{1 / 2}$ ionic state (Fig. 3). The $f$-resonance cannot be identified in the polarization curve which indicates that the noninteracting continuum is unpolarized.

\section{Conclusion}

The first spin-polarization measurements of autoionizing levels of mercury have been performed in the range between the $\mathrm{Hg}^{+}\left(5 d^{9} 6 s^{2}\right)^{2} D$-thresholds where the autoionizing levels can decay into partial continua corresponding to two final $\left[5 d^{9} 6 s^{2}\left({ }^{2} D_{3 / 2}\right) \quad n p_{1 / 2}, n p_{3 / 2}, n f_{5 / 2}\right]_{J=1}$
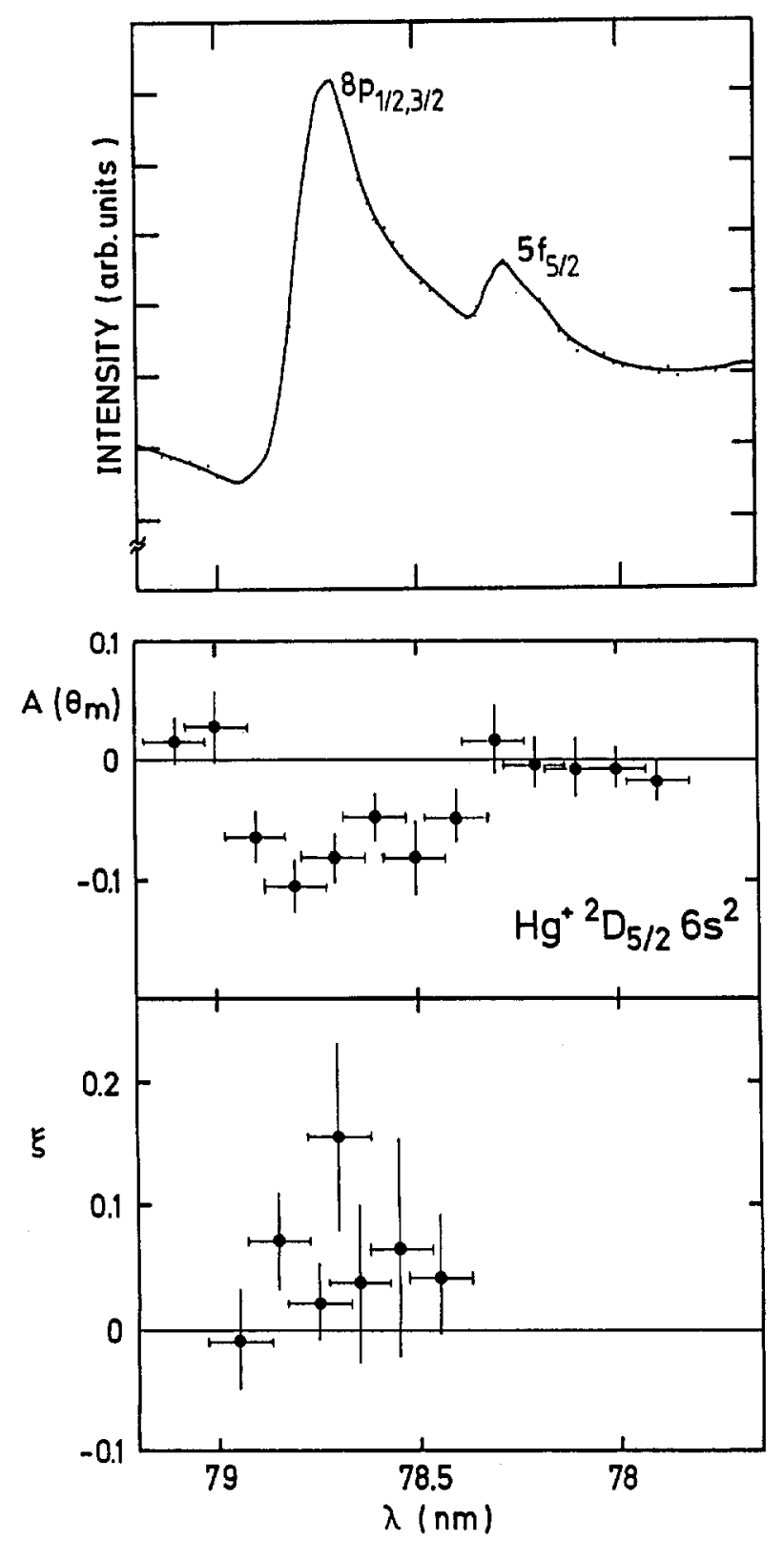

Fig. 4. Photoelectron intensity and spin-polarization parameters $A$ and $\xi$ as a function of the wavelength in the range of the $\mathrm{Hg}^{*}\left(5 d^{9} 6 s^{2}\right)^{2} D_{3 / 2} 8 p_{1 / 2}, p_{3 / 2}$ and $5 f_{5 / 2}$ resonance for $\mathrm{Hg}^{+2} D_{5 / 2}$.

ionic states. The photoelectron intensity spectra and the spin-polarization data have revealed significant differences of the coupling of the autoionizing levels to the two final ionic states. Moreover, new resonances with complementary coupling to both ionic channels have been observed. Further analysis of the data which should lead to a decoupling of all partial continua and an evaluation of matrix elements and phase-shift differences is in progress.

\section{Acknowledgement}

We thank the BESSY staff for fruitful cooperation and acknowledge support from the Bundesministerium für Forschung und Technologie (05331AX).

\section{References}

1. Beutler, J., Z. Phys. 86, 710 (1933). 
2. Garton, W. R. S. and Connerade, J. P., Astrophys. J. 155, 667 (1969).

3. Mansfield, M. W. D., Astrophys. J. 180, 1011 (1973)

4. Brehm, B., Z. Naturf. 21a, 196 (1966).

5. Cairns, R. B., Harrison, H. and Schoen, R. I., J. Chem. Phys. 53, 96 (1970).

6. Berkowitz, J., Photoabsorption, Photoionisation and Photoelectron Spectroscopy, Academic Press, New York, 1979.

7. Blake, A. J., Proc. R. Soc. Lond. A325, 555 (1971)

8. Brehm, B. and Höfler, K., Phys. Lett. 68a, 437 (1978).

9. Schäfers, F., Schönhense, G. and Heinzmann, U., Z. Phys. A 304, 41 (1982).

10. Schönhense, G., Schäfers, F., Heckenkamp, Ch., Heinzmann, U. and Baig, M. A., J. Phys. B: At. Mol. Phys. 17, L771 (1984).
11. Bartschat, K. and Scott, P., J. Phys. B: At. Mol. Phys. 18, L191 (1985).

12. Bartschat, K. and Scott, P., J. Phys. B: At. Mol. Phys. 18, 3725 (1985).

13. Heckenkamp, Ch., Schäfers, F., Schönhense, G. and Heinzmann, U., Phys. Rev. Lett. 52, 421 (1984).

14. Heckenkamp, Ch., Schäfers, F., Schönhense, G. and Heinzmann, U., Z. Phys. D. 2, 257 (1986)

15. Heckenkamp, Ch., Eyers, A., Schäfers, F., Schönhense, G. and Heinzmann, U., Nucl. Instrum. Methods A246, 500 (1986).

16. Schäfers, F., Peatman, W., Eyers, A., Heckenkamp, Ch., Schönhense, G. and Heinzmann, U., Rev. Sci. Instr. 57, 1032 (1986).

17. Fano, U., Phys. Rev. 124, 1866 (1961).

18. Krause, M. O., Cerrina, F,, Fahlman, A. and Carlson, Th. A., Phys. Rev. Lett. 51, 2093 (1983). 